\title{
An analysis of the implicit choice set generation using the Constrained Multinomial Logit model
}

Michel Bierlaire (corresponding author)

Transport and Mobility Laboratory

Ecole Polytechnique Fédérale de Lausanne

Lausanne 1015, Switzerland

Tel: +41.21 .6932537$

Fax: +41.21 .6938060$

Email: michel.bierlaire@epfl.ch

\section{Ricardo Hurtubia}

Transport and Mobility Laboratory

Ecole Polytechnique Fédérale de Lausanne

Lausanne 1015, Switzerland

Tel: +41.21.6939329

Fax: +41.21 .6938060$

Email: ricardo.hurtubia@epfl.ch

\section{Gunnar Flötteröd}

Transport and Mobility Laboratory

Ecole Polytechnique Fédérale de Lausanne

Lausanne 1015, Switzerland

Tel: +41.21.6932429

Fax: +41.21 .6938060$

Email: gunnar.floetteroed@epfl.ch

$$
3945 \text { words }+7 \text { figures }+3 \text { tables }
$$

November 15, 2009 


\begin{abstract}
Discrete choice models are defined conditional to the knowledge of the actual choice set by the analyst. The common practice for many years is to assume that individual-based choice sets can be deterministically generated based on the choice context and the characteristics of the decision maker. There are many situations where this assumption is not valid or not applicable, and probabilistic choice set formation procedures must be considered.

The Constrained Multinomial Logit model (CMNL) has recently been proposed by Martinez et al. (1) as a convenient way to deal with this issue, as it is also appropriate for models with a large choice set. In this paper, we analyze how well the implicit choice set generation of the CMNL approximates to the explicit choice set generation as described by Manski (2).

The results based on synthetic data show that the implicit choice set generation model may be a poor approximation of the explicit model.
\end{abstract}




\section{INTRODUCTION}

In standard choice models, it is assumed that the alternatives considered by the decision maker can be deterministically specified by the analyst. The choice set is characterized by deterministic rules based on the characteristics of the decision maker and the choice context. For example, singleroom apartments are not considered by families with children in a house choice context, car is not considered as a possible transportation mode if the traveler has no driver license, or no car.

There are, however, many situations where the deterministic choice set generation procedure is not satisfactory, or even possible. Data may be unavailable (the number of children in the household is unknown to the analyst), or rules are fuzzy by nature. For instance, train is not considered as a transportation mode if it involves a long walk to reach the train station. But how long is a "long walk"?

Modeling explicitly the choice set generation process involves a combinatorial complexity, which makes the models intractable except for some specific instances. Manski (2) defines the theoretical framework in a two stage process, where the probability that decision maker $n$ chooses alternative $i$ is given by

$$
P_{n}(i)=\sum_{\mathcal{C}_{m} \subseteq \mathcal{C}} P_{n}\left(i \mid \mathcal{C}_{m}\right) P_{n}\left(\mathcal{C}_{m}\right)
$$

where $P_{n}\left(i \mid \mathcal{C}_{m}\right)$ is the probability for individual $n$ to choose alternative $i$ conditional to the choice set $\mathcal{C}_{m}$ and $P_{n}\left(\mathcal{C}_{m}\right)$ is the probability for individual $n$ to consider choice set $\mathcal{C}_{m}$. The sum runs on every possible subset $\mathcal{C}_{m}$ of the universal choice set $\mathcal{C}$.

Swait and Ben-Akiva (3) and Ben-Akiva and Boccara (4) build on this framework and use explicit random constraints to determine the choice set generation probability. The probability of considering a choice set $\mathcal{C}_{m}$ is a function of the consideration of the different alternatives in the universal choice set:

$$
P_{n}\left(\mathcal{C}_{m}\right)=\frac{\prod_{i \in \mathcal{C}_{m}} \phi_{i n} \prod_{j \notin \mathcal{C}_{m}}\left(1-\phi_{j n}\right)}{1-\prod_{k \in \mathcal{C}}\left(1-\phi_{k n}\right)}
$$

where $\phi_{i n}$ is the probability that alternative $i$ is considered by user $n$, which may be modeled by a binary logit model that depends on the alternative's attributes. Note that (2) assumes independence of the consideration probabilities across alternatives, which is a restrictive assumption since there can be correlation in the consideration criteria of different alternatives.

Swait (5) proposes to model the choice set generation as an implicit part of the choice process in a multivariate extreme value (MEV) framework, requiring no exogenous information. Here, choice sets are not separate constructs but another expression of preferences. The probability of considering a choice set is defined as the probability for that choice set to correspond to the maximum expected utility for an individual $n$ :

$$
P_{n}\left(\mathcal{C}_{m}\right)=\frac{e^{\mu I_{n, \mathcal{C}_{m}}}}{\sum_{\mathcal{C}_{k} \subseteq \mathcal{C}} e^{\mu I_{n, \mathcal{C}_{k}}}}
$$

where $\mu$ is the scale parameter for the higher level decision (choice set selection) and $I_{n, \mathcal{C}_{m}}$ is the inclusive value (the "logsum" or expected maximum utility) of choice set $\mathcal{C}_{m}$ for decision maker $n$ :

$$
I_{n, \mathcal{C}_{m}}=\frac{1}{\mu_{m}} \ln \sum_{j \in \mathcal{C}_{m}} e^{\mu_{m} V_{n j}} .
$$


Here, $\mu_{m}$ is the scale parameter and $V_{n j}$ is the deterministic utility of alternative $i$ for decision maker $n$. Swait's probabilistic choice set generation approach does not require assumptions by the analyst about which attributes affect an alternative's availability. Note that Swait's model also needs to account for every possible subset $\mathcal{C}_{m}$ of the universal choice set $\mathcal{C}$.

Clearly, these methods are hardly applicable to medium to large scale choice problems due to the computational complexity that arises from the combinatorial number of possible choice sets. If the number of alternatives in the universal choice set is $J$, the number of possible choice sets is $\left(2^{J}-1\right)$.

In the context of route choice, Frejinger et al. (6) assume that all decision makers consider the universal choice set, so that $P_{n}\left(\mathcal{C}_{m}\right)=0$ when $\mathcal{C}_{m} \neq \mathcal{C}$, and only one term remains in (1). However, this may not be appropriate in other contexts.

Therefore, various heuristics have been proposed in the literature that derive tractable models by approximating the choice set generation process.

In the quantitative marketing literature, the use of heuristics to model the construction of the choice set (or consideration set) has been a usual practice; a review of existing models can be found in Hauser et al. (7). Many heuristics are based on lexicographic preferences rules (Dieckmann et al. (8)), where the choice set is determined by key attributes of the alternatives on which the consumers base the construction of their consideration set. This approach is similar to the elimination by aspects heuristic, proposed by Tversky (9). Models like the one proposed by Gilbride and Allenby (10) consider the construction of the choice set as a two-stage process, which is consistent with Manski's approach but solves the choice set enumeration issue by using Bayesian and Monte Carlo estimation methods.

Other heuristics use a one stage approach (see for example Elrod et al. (11)) where the choice set generation process is simulated through direct alternative elimination. This is done by setting the alternative's utility to minus infinity when certain attributes reach a threshold value. The alternative-elimination approach implies a different behavioral assumption from the two-stage approach, where the individual does not observe choice sets explicitly but, instead, makes a compensatory choice between all the alternatives belonging to a unique choice set of available or "possible" alternatives, which is a sub-set of the universal choice set.

Following the same one-stage approach, other heuristics assume that the elimination of the alternatives is not deterministic. These are based on the use of penalties in the utility functions, and have been proposed by Cascetta and Papola (12) (the Implicit Availability/Perception (IAP) model) and expanded by Martinez et al. (1) (the Constrained Multinomial Logit or CMNL model). In the next section, we briefly describe the CMNL model and provide its theoretical background in the context of choice set generation. In Section 3, we compare the CMNL with the theoretical framework (1), first through a simple example and, second, by estimating both models on synthetic data. Section 4 concludes the paper and identifies possible further work.

\section{CHOICE SET GENERATION WITH THE CMNL MODEL}

Assuming that $\mathcal{C}_{n}$ is the choice set that the decision maker is actually considering, the choice model is given by

$$
P_{n}\left(i \mid \mathcal{C}_{n}\right)=\operatorname{Pr}\left(U_{i n} \geq U_{j n}, \forall j \in \mathcal{C}_{n}\right),
$$

where $U_{i n}$ is the random utility associated with alternative $i$ by decision maker $n$. If $\mathcal{C}_{n}$ is known to the analyst, it can be characterized by indicators of the consideration of each alternative by the 
decision maker:

$$
A_{i n}= \begin{cases}1 & \text { if alternative } i \text { is considered by individual } n \\ 0 & \text { otherwise. }\end{cases}
$$

The choice model can be equivalently written as

$$
\begin{aligned}
P_{n}\left(i \mid \mathcal{C}_{n}\right) & =\operatorname{Pr}\left(U_{i n} \geq U_{j n}, \forall j \in \mathcal{C}_{n}\right) \\
& =\operatorname{Pr}\left(U_{i n}+\ln A_{i n} \geq U_{j n}+\ln A_{j n}, \forall j \in \mathcal{C}\right)
\end{aligned}
$$

For an unconsidered alternative, this adds $\ln 0=-\infty$ to its utility, so that the choice probability is 0 , whereas the addition of $\ln 1=0$ has no effect on the utility of a considered alternative.

In the case of a logit model, the choice probabilities are

$$
P_{n}(i)=\frac{e^{V_{i n}+\ln A_{i n}}}{\sum_{j \in C} e^{V_{j n}+\ln A_{j n}}} .
$$

The heuristics proposed by Cascetta and Papola (12) and Martinez et al. (1) consist in replacing the indicators $A_{i n}$ by the probability $\phi_{i n}$ that individual $n$ considers alternative $i$.

Cascetta and Papola (12) introduce the IAP model as a way to incorporate awareness of paths into route choice modeling without requiring an explicit choice set generation step. A similar approach that penalizes the utilities of "dominated" alternatives is proposed by Cascetta et al. (13).

Martinez et al. (1) expand the IAP idea and propose the CMNL model. The functional form for $\phi_{i n}$ is assumed to be a binary logit, considering that the availability of an alternative is related with bound constraints on its attributes. For example, if $X_{i n k}$ is the $k$ th variable of alternative $i$ for decision maker $n$ that influences the consideration of $i$, we have

$$
\phi_{i n}^{u}\left(X_{i n k} ; u_{k}, \omega_{k}\right)=\frac{1}{1+\exp \left(\omega_{k}\left(X_{i n k}-u_{k}\right)\right)}
$$

where the $u_{k}$ parameter is the value at which the constraint is most likely to bind, and $\omega_{k}$ is the scale parameter of the binary logit. For instance, $X_{i n k}$ may be the walking distance to the train station, and $u_{k}$ may be the maximum distance that individual $n$ is willing to walk. Both $u_{k}$ and $\omega_{k}$ are to be estimated. The intuition is that when the attribute $X_{\text {ink }}$ exceeds $u_{k}$, the consideration probability $\phi_{i n}^{u}$ tends to zero, while this availability tends to one when the value of the attribute is below $u_{k}$.

Expression (9) represents an upper value cut-off, where $u_{k}$ represents the maximum value that the attribute $X_{\text {ink }}$ can have in order for alternative $i$ to be considered. To model a lower value cut-off, we only need to invert the sign of the scale parameter $\omega_{k}$ :

$$
\phi_{i n}^{\ell}\left(X_{i n k} ; \ell_{k}, \omega_{k}\right)=\frac{1}{1+\exp \left(-\omega_{k}\left(X_{i n k}-\ell_{k}\right)\right)} .
$$

Functions (9) and (10) can be generalized to account for more than one constraint, allowing for several upper and lower bounds to be included simultaneously:

$$
\phi_{i n}\left(X_{i n} ; \ell, u, \omega\right)=\prod_{k} \phi_{i n}^{u}\left(X_{i n k} ; u_{k}, \omega_{k}\right) \phi_{i n}^{\ell}\left(X_{i n k} ; \ell_{k}, \omega_{k}\right)
$$


The CMNL approach has an operational advantage over Manski's framework since it does not require enumerating the choice sets, which makes it easier to specify and estimate. However, the CMNL model is a heuristic that is based on convenient assumptions about the functional form of the utility function. This is why the CMNL model can at most be considered as an approximation to Manski's model. The next section evaluates the quality of this approximation.

\section{COMPARISON OF CMNL WITH MANSKI'S MODEL}

This section compares the CMNL model with Manski's model. For this, we first present a simple example where we analyze the difference between the choice probabilities obtained using both models. Second, we estimate the CMNL model and Manski's model over synthetic data and compare the results. For notational simplicity, we subsequently omit the index $n$ for the decision maker.

\subsection{Simple example}

Consider a logit model with only 2 alternatives, where alternative 1 is always considered $\left(\phi_{1}=1\right)$ and alternative 2 has probability $\phi_{2}$ of being considered by the decision maker. Figure 1 shows the structure of Manski's framework if we consider every possible combination of alternatives as a choice set. This simple situation corresponds to a case where the decision maker is captive to alternative 1 with probability $1-\phi_{2}$ (see also the captivity logit model proposed by Gaudry and Dagenais (14)).

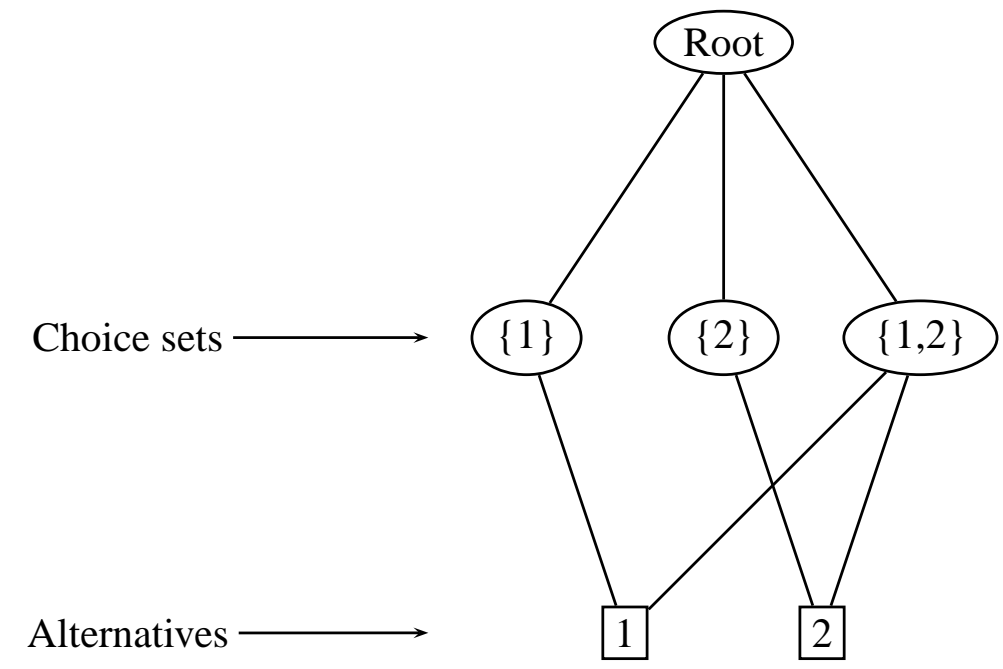

\section{FIGURE 1 Example of a model in Manski's framework}

The CMNL model defines the probability of choosing alternative 1 as

$$
P(1)=\frac{e^{V_{1}}}{e^{V_{1}}+e^{V_{2}+\ln \phi_{2}}} .
$$


Manski's model (1) defines the probability of choosing alternative 1 as

$$
P(1)=P(\{1\}) \frac{e^{V_{1}}}{e^{V_{1}}}+P(\{1,2\}) \frac{e^{V_{1}}}{e^{V_{1}}+e^{V_{2}}}
$$

where $P(\{1\})$ is the probability of considering the choice set composed only of alternative 1 and $P(\{1,2\})$ is the probability of considering the choice set containing both alternatives. According to (2), the choice set probabilities are

$$
P(\{1\})=\frac{\phi_{1}\left(1-\phi_{2}\right)}{1-\left(1-\phi_{1}\right)\left(1-\phi_{2}\right)}=1-\phi_{2}
$$

and

$$
P(\{1,2\})=\frac{\phi_{1} \phi_{2}}{1-\left(1-\phi_{1}\right)\left(1-\phi_{2}\right)}=\phi_{2}
$$

The probability of considering choice set $\{2\}$ is zero because alternative 1 is always available. Therefore, (13) becomes

$$
P(1)=\left(1-\phi_{2}\right)+\phi_{2} \frac{e^{V_{1}}}{e^{V_{1}}+e^{V_{2}}}
$$

In the deterministic limit $\left(\phi_{2}=0\right.$ or $\phi_{2}=1$ ), both models are equivalent. However, this is not the case anymore when $\phi_{2}$ takes values between zero and one. The resulting choice probabilities are shown in Figure 2, assuming the same utility level $V_{1}=V_{2}$ for both alternatives.

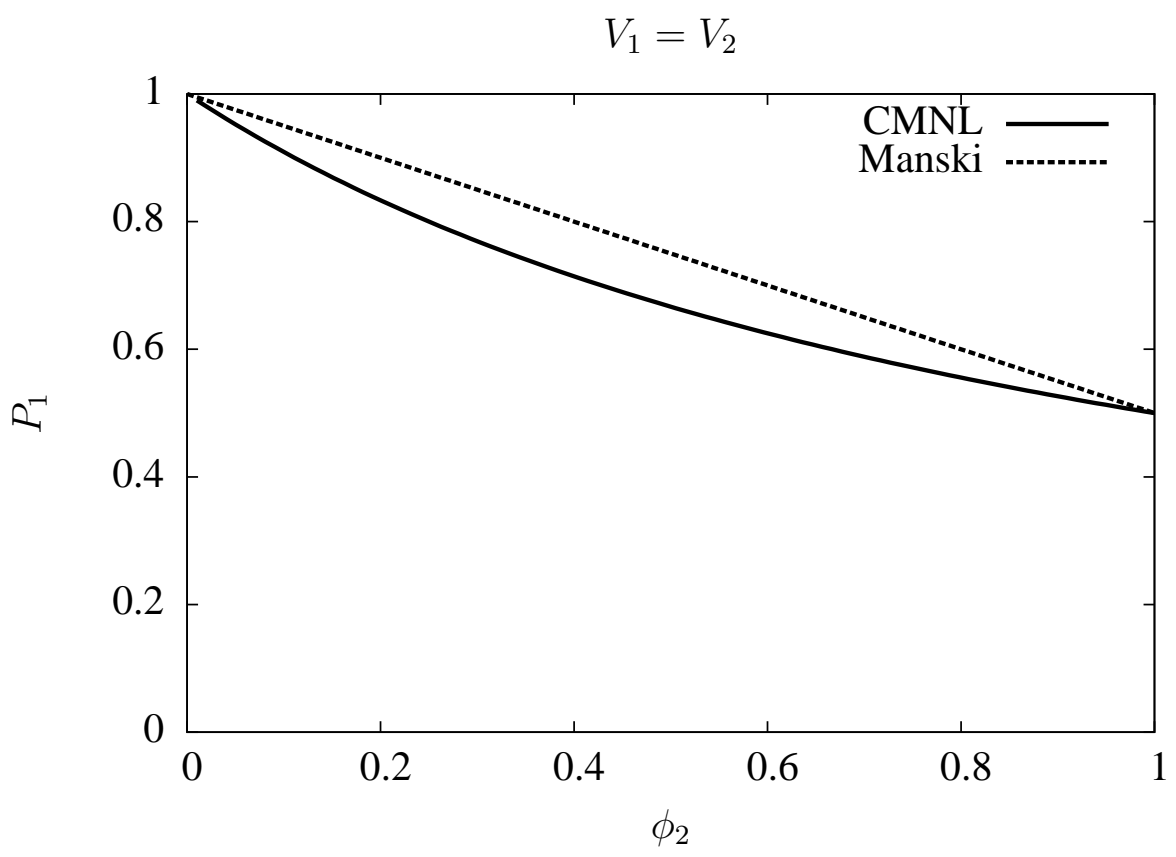

FIGURE 2 Choice probability of alternative $1\left(V_{1}=V_{2}\right)$ 
This figure shows that the CMNL is a good approximation of Manski's model only when $\phi_{2}$ is close to either zero or one, but it underestimates the probability of alternative 1 elsewhere. If the utility for alternative 1 is larger than the utility for alternative 2 (Figure 3), the approximation improves. This makes sense since the more an alternative is dominated, the less important it is to know if it really belongs to the choice set.

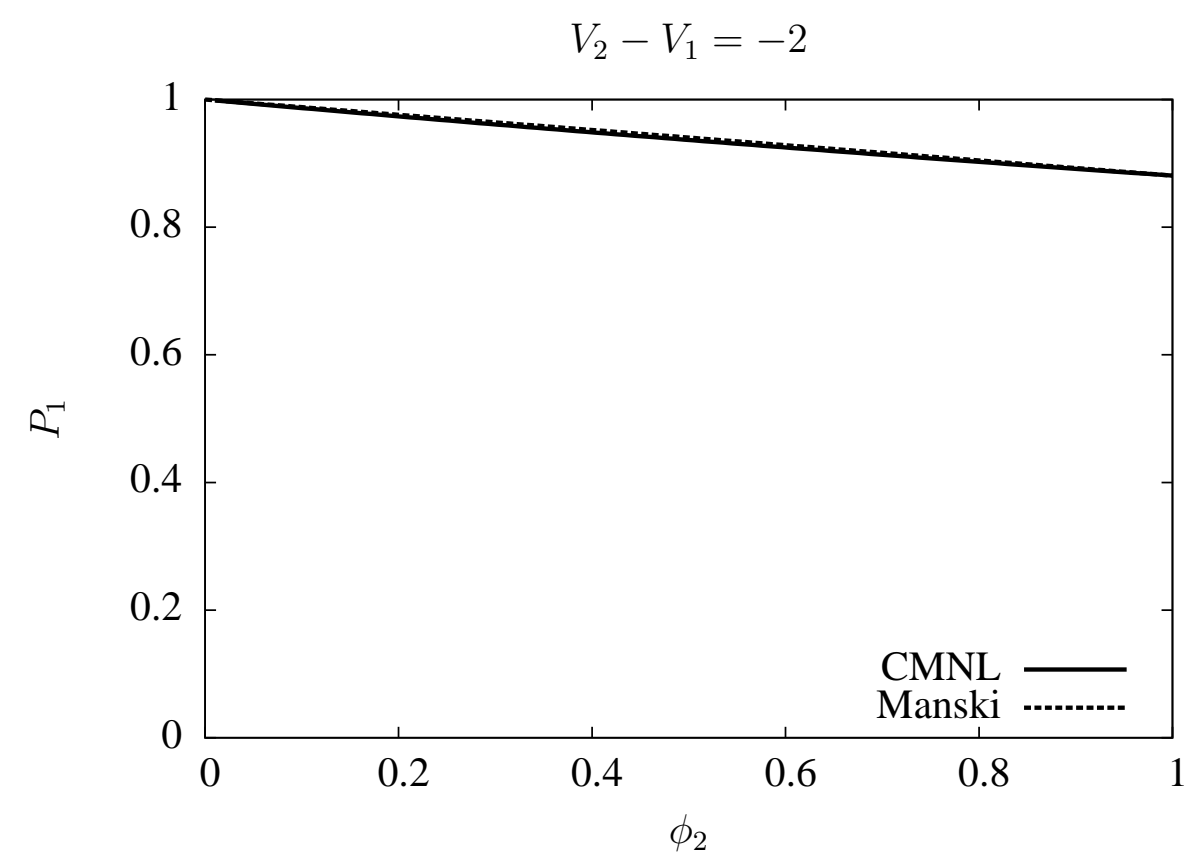

\section{FIGURE 3 Probability of alternative $1\left(V_{1}>V_{2}\right)$}

However, as the utility of alternative 1 becomes smaller and smaller compared to the utility of alternative 2, the CMNL becomes a poorer and poorer approximation of Manski's model for intermediate $\phi_{2}$ values, which is demonstrated in Figures 4 and 5.

These results can be interpreted as an unwanted compensatory effect in the CMNL model. The availability constraint is enforced by modifying the utility of the constrained alternative. However, when the utility of this alternative is high, it compensates the penalty. This means that the use of the CMNL model as an efficient choice set generation mechanism requires the assumption that the consideration probability for an alternative grows with its utility, meaning that the choice set depends only on the preferences of the individual. But alternatives with a high utility may be discarded in the presence of constraints such as budget or physical constraints. In the context of repetitive choices over a long period the individual may try to change her initial constraints in order to make the high-utility alternative available (for example, if the train produces high utility, a user may consider moving his residence closer to the train station), but in an instantaneous or short-term decision this may not be possible. This motivates to analyze the performance of the CMNL on synthetic data, which is shown in the next section. 


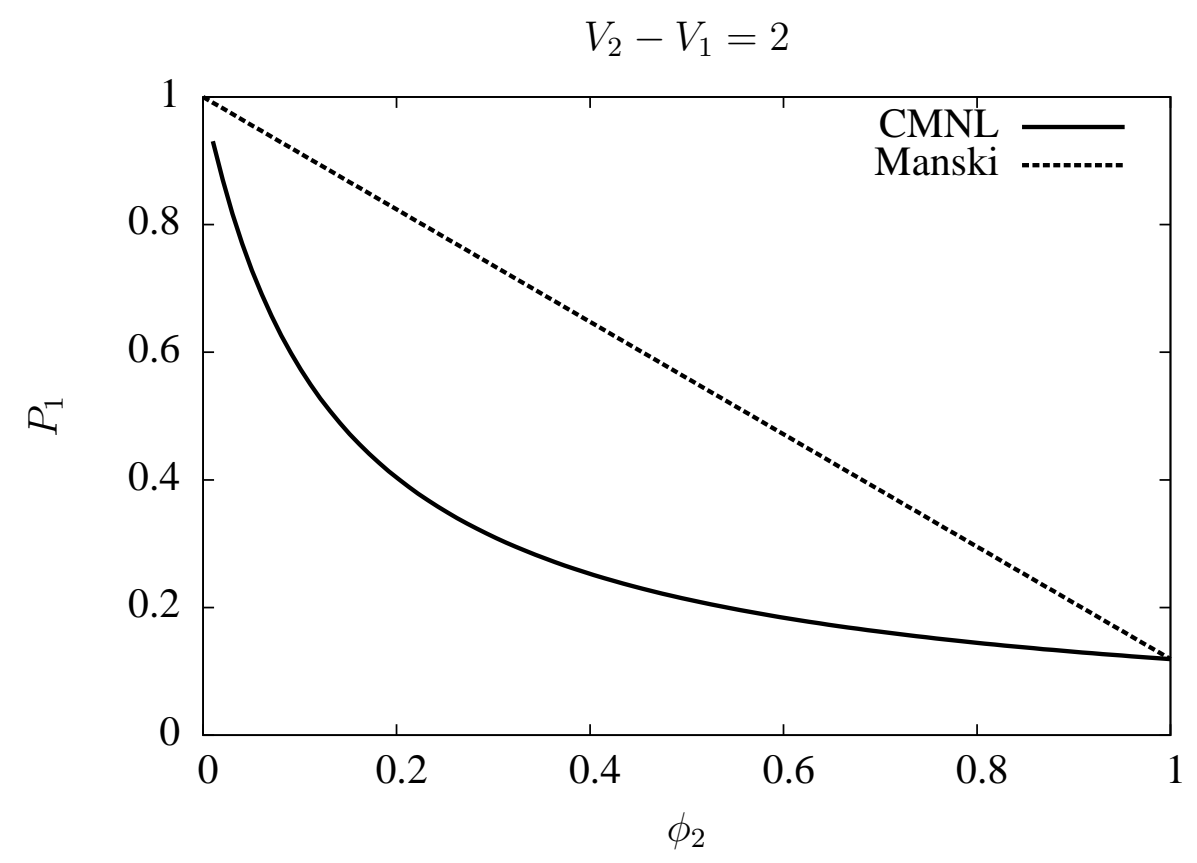

FIGURE 4 Choice probability of alternative $1\left(V_{1}<V_{2}\right)$

\subsection{Synthetic data}

This section describes a series of controlled experiments where some of the data is synthetically generated. We start from a real stated preference data set that was collected for the analysis of a hypothetical high speed train in Switzerland (Bierlaire et al. (15)). The alternatives are:

\section{Driving a car (CAR)}

\section{Regular train (TRAIN)}

3. Swissmetro, the future high speed train (SM)

From this data set, which consists of 5607 observations, we use the attributes of the alternatives and simulate synthetic choices based on a postulated "true" model: a logit model with linear-inparameters utility functions. The specification table as well as the "true" values of the parameters are reported in Table 1 . The values have been obtained by estimating the model on real choices, and by rounding the estimates.

It is assumed that the TRAIN and the SM alternatives are always considered, whereas the consideration of the CAR alternative depends on the travel time according to

$$
\phi_{\mathrm{CAR}}=\frac{1}{1+\exp \left(\omega\left(T T_{\mathrm{CAR}} / 60-a\right)\right)},
$$

which states that the probability of considering CAR as an available alternative decreases with the travel time $T T_{C A R}$, in minutes, and that this probability is 0.5 when the availability threshold $a$, in hours, is reached. 


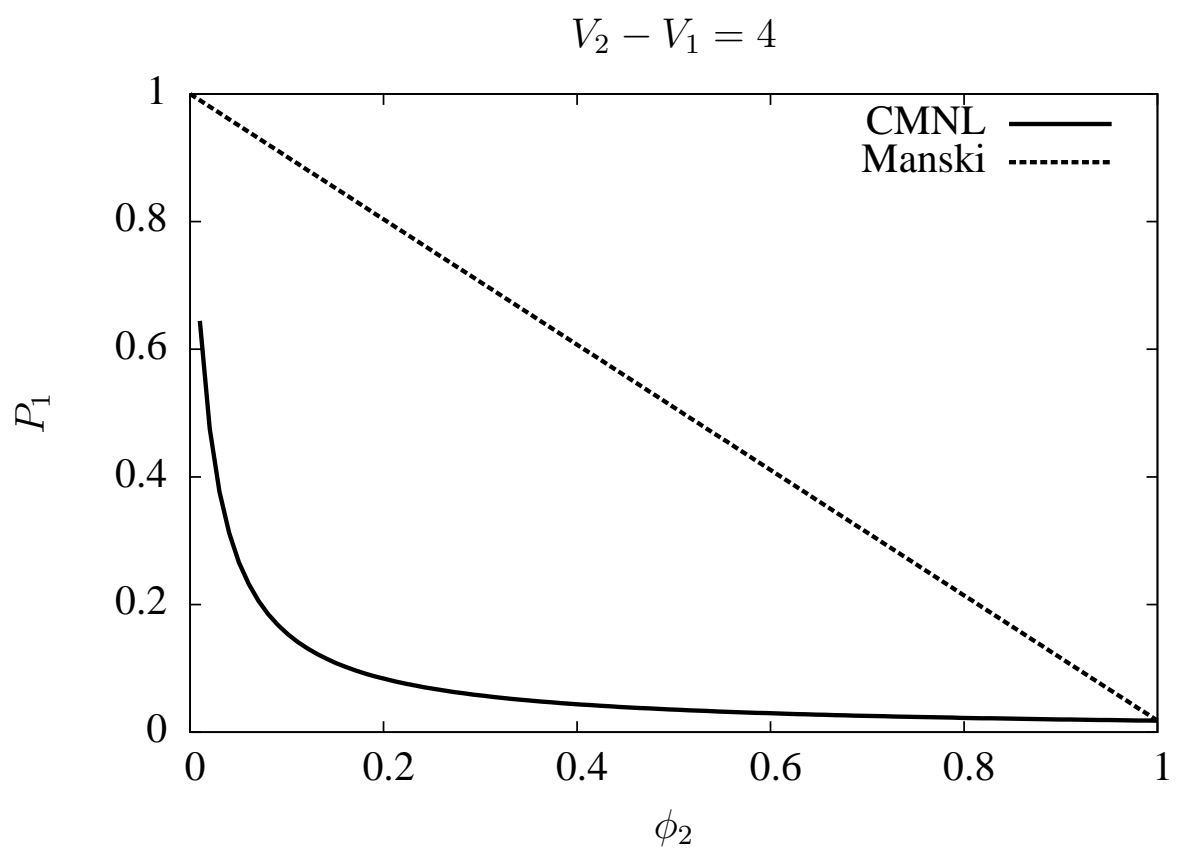

FIGURE 5 Choice probability of alternative $1\left(V_{1}<V_{2}\right)$

This implies that, depending on the availability of the CAR alternative, there are two possible choice sets: the full choice set and the choice set containing only the TRAIN and the SM alternative. The random constraints approach (Ben-Akiva and Boccara (4)) defines the probability of each choice set as follows:

$$
\begin{aligned}
P(\{\text { TRAIN }, \mathrm{SM}\}) & =\frac{\phi_{\mathrm{TRAIN}} \phi_{\mathrm{SM}}\left(1-\phi_{\mathrm{CAR}}\right)}{1-\left(1-\phi_{\mathrm{CAR}}\right)\left(1-\phi_{\mathrm{TRAIN}}\right)\left(1-\phi_{\mathrm{SM}}\right)} \\
& =1-\phi_{\mathrm{CAR}}
\end{aligned}
$$

and, accordingly,

$$
P(\{\mathrm{CAR}, \mathrm{TRAIN}, \mathrm{SM}\})=\phi_{\mathrm{CAR}} .
$$

The synthetic choices are generated by (i) simulating a choice set for each decision maker according to (18) and (19), and (ii) simulating a choice for each decision maker using the "true" model specified in Table 1.

100 choice data sets are simulated for each value of $\omega$. These values generate constraints with different levels of uncertainty. Figure 6 shows the shape of these constraint functions. Estimation results for both the Manski and the CMNL model are given in Tables 2 and 3. For each parameter $\beta$, the average value $\bar{\beta}$ and the standard error $\sigma$ over 100 simulations are computed. In the tables, both $\bar{\beta}$ and the t-statistic $(\bar{\beta}-\beta) / \sigma$ are reported, the latter value being used to test if the estimated value is significantly different from the true one. Note that, since the tested hypothesis is that the average estimated value is equal to the "true" one, a low value of the t-statistic indicates that the estimate is not significantly different from the real parameter.

The estimates of Manski's model are unbiased. We cannot reject the hypothesis that the true value of any parameters is equal to the postulated value, at $95 \%$ level. Several estimates of 
TABLE 1 Parameter descriptions and values

\begin{tabular}{l|l|lll} 
Parameter & Value & Car & Train & Swissmetro \\
\hline ASC $_{\text {CAR }}$ & 0.3 & 1 & 0 & 0 \\
ASC $_{\text {SM }}$ & 0.4 & 0 & 0 & 1 \\
$\beta_{\text {cost }}$ & -0.001 & Cost (CHF) & Cost (CHF) & Cost (CHF) \\
$\beta_{t t}$ & -0.001 & In veh. travel time (min- & In veh. travel time (min- & In veh. travel time (min- \\
& & utes) & utes) & utes) \\
$\beta_{h e}$ & -0.005 & 0 & Headway (minutes) & Headway (minutes) \\
\hline$a$ & 3 & Consideration threshold of car (hours) & \\
$\omega$ & $1,2,3,5,10$ & Consideration dispersion of car &
\end{tabular}

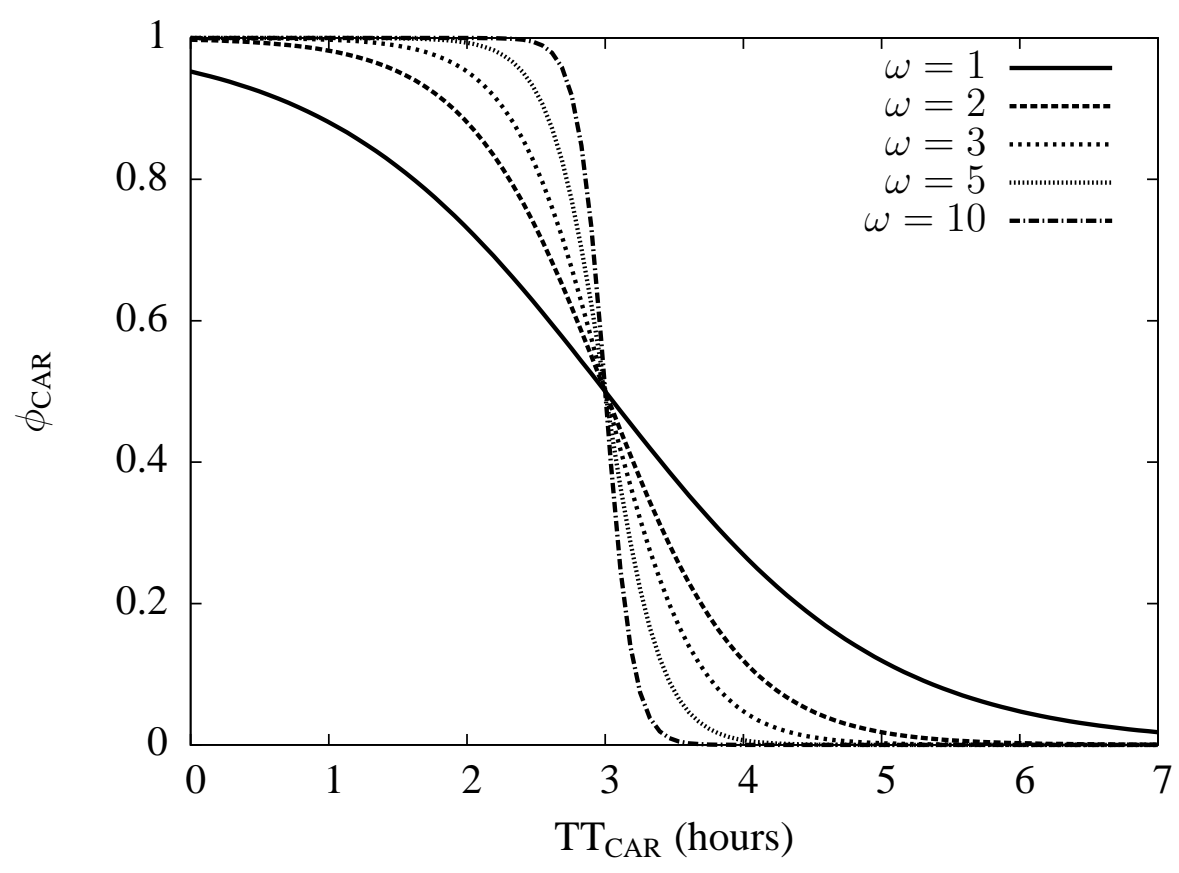

FIGURE 6 Shape of the constraint for different values of $\boldsymbol{\omega}$ 
TABLE 2 Estimation results for Manski's model

\begin{tabular}{|c|c|c|c|l|l|l|l|l|l|l|l|}
\hline \multicolumn{2}{|c|}{ real $\omega$ value } & \multicolumn{2}{l|}{1} & \multicolumn{2}{l|}{3} & \multicolumn{2}{l|}{5} & \multicolumn{2}{l|}{10} \\
\hline parameter & real value & estimate & $\mathrm{t}$-test & estimate & $\mathrm{t}$-test & estimate & $\mathrm{t}$-test & estimate & $\mathrm{t}$-test & estimate & $\mathrm{t}$-test \\
\hline$A S C_{\mathrm{CAR}}$ & 0.3 & 0.304 & 0.027 & 0.288 & 0.113 & 0.300 & 0.010 & 0.301 & 0.012 & 0.314 & 0.184 \\
\hline$A S C_{\mathrm{SM}}$ & 0.4 & 0.396 & 0.044 & 0.399 & 0.010 & 0.405 & 0.053 & 0.401 & 0.017 & 0.410 & 0.151 \\
\hline$\beta_{\text {cost }}$ & -0.01 & -0.010 & 0.283 & -0.010 & 0.001 & -0.010 & 0.179 & -0.010 & 0.052 & -0.010 & 0.012 \\
\hline$\beta_{\text {he }}$ & -0.005 & -0.005 & 0.241 & -0.005 & 0.010 & -0.005 & 0.048 & -0.005 & 0.082 & -0.005 & 0.078 \\
\hline$\beta_{\text {time }}$ & -0.01 & -0.01 & 0.074 & -0.010 & 0.050 & -0.010 & 0.049 & -0.010 & 0.003 & -0.010 & 0.001 \\
\hline$a$ & 3 & 2.963 & 0.019 & 3.008 & 0.118 & 3.000 & 0.100 & 2.998 & 0.081 & 3.002 & 0.101 \\
\hline$\omega$ & see top & 1.003 & 0.028 & 2.014 & 0.079 & 3.066 & 0.210 & 5.095 & 0.170 & 10.523 & 0.353 \\
\hline
\end{tabular}


TABLE 3 Estimation results for CMNL model

\begin{tabular}{|c|c|c|c|c|c|l|c|c|c|c|c|}
\hline \multicolumn{2}{|c|}{ real $\omega$ value } & \multicolumn{2}{l|}{ l } & \multicolumn{3}{l|}{3} & \multicolumn{2}{l|}{5} & 10 \\
\hline parameter & real value & estimate & t-test & estimate & t-test & estimate & t-test & estimate & t-test & estimate & t-test \\
\hline$A S C_{\text {CAR }}$ & 0.3 & 0.503 & 0.950 & 0.421 & 1.153 & 0.406 & 1.365 & 0.380 & 0.988 & 0.326 & 0.313 \\
\hline$A S C_{\text {SM }}$ & 0.4 & 0.565 & $2.013 *$ & 0.550 & $2.375 *$ & 0.536 & 1.804 & 0.506 & 1.485 & 0.463 & 0.872 \\
\hline$\beta_{\text {cost }}$ & -0.01 & -0.008 & $4.825 *$ & -0.008 & $3.580 *$ & -0.009 & $2.309 *$ & -0.009 & 1.182 & -0.010 & 0.613 \\
\hline$\beta_{\text {he }}$ & -0.005 & -0.005 & 0.202 & -0.005 & 0.151 & -0.005 & 0.071 & -0.005 & 0.120 & -0.005 & 0.090 \\
\hline$\beta_{\text {time }}$ & -0.01 & -0.007 & $3.929 *$ & -0.008 & $3.645 *$ & -0.008 & $2.813 *$ & -0.009 & $2.316 *$ & -0.009 & 1.523 \\
\hline$a$ & 3 & 2.186 & 1.753 & 2.656 & $3.073 *$ & 2.773 & $3.762 *$ & -2.869 & $3.305 *$ & 2.948 & 1.864 \\
\hline$\omega$ & see top & 1.043 & 0.239 & 2.094 & 0.403 & 3.118 & 0.431 & 5.238 & 0.424 & 12.146 & $3.149 *$ \\
\hline
\end{tabular}

(* indicates a biased parameter) 
the CMNL model are biased (marked with *), the hypothesis that the true value of the parameter is equal to the postulated value being rejected at the $95 \%$ level. The quality of the CMNL estimates improves with decreasing dispersion (increasing $\omega$ ). This is consistent with the findings of Section 3.1 .

Figure 7 shows the t-statistics for the cost and travel time parameter over different $\omega$ values for Manski's model and the CMNL model. The quality of the estimates is constant across different values of $\omega$ for Manski's model. The quality of the CMNL estimates increases with $\omega$, and their t-statistics reach acceptable values when the constraint function becomes steep.

\section{CONCLUSIONS AND FURTHER WORK}

We have shown on simple examples that the Constrained Multinomial Logit (CMNL) model is not adequate to model the choice set generation process consistently with Manski's framework. Consequently, the CMNL model should be considered as a model on its own, derived from semicompensatory assumptions as described by Martinez et al. (1), but not as a way to capture the choice set generation process. Its complexity is linear with the number of alternatives, while Manski's framework exhibits an exponential complexity.

We have started to investigate if a modified version of the CMNL could approximate better Manski's framework, but have been unsuccessful so far. The derivation of a good approximation of Manski's model with the complexity of the CMNL would be particularly useful to handle models with a large number of alternatives. 


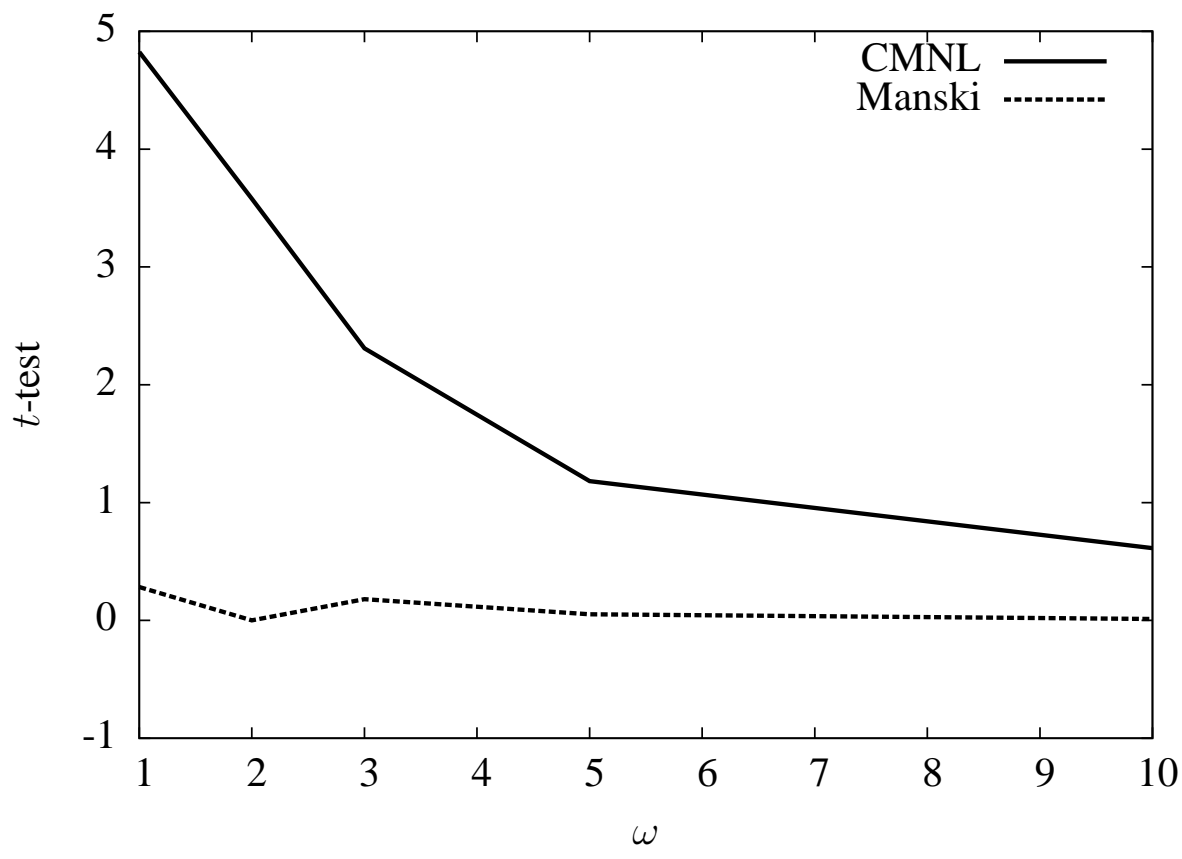

(a) Cost

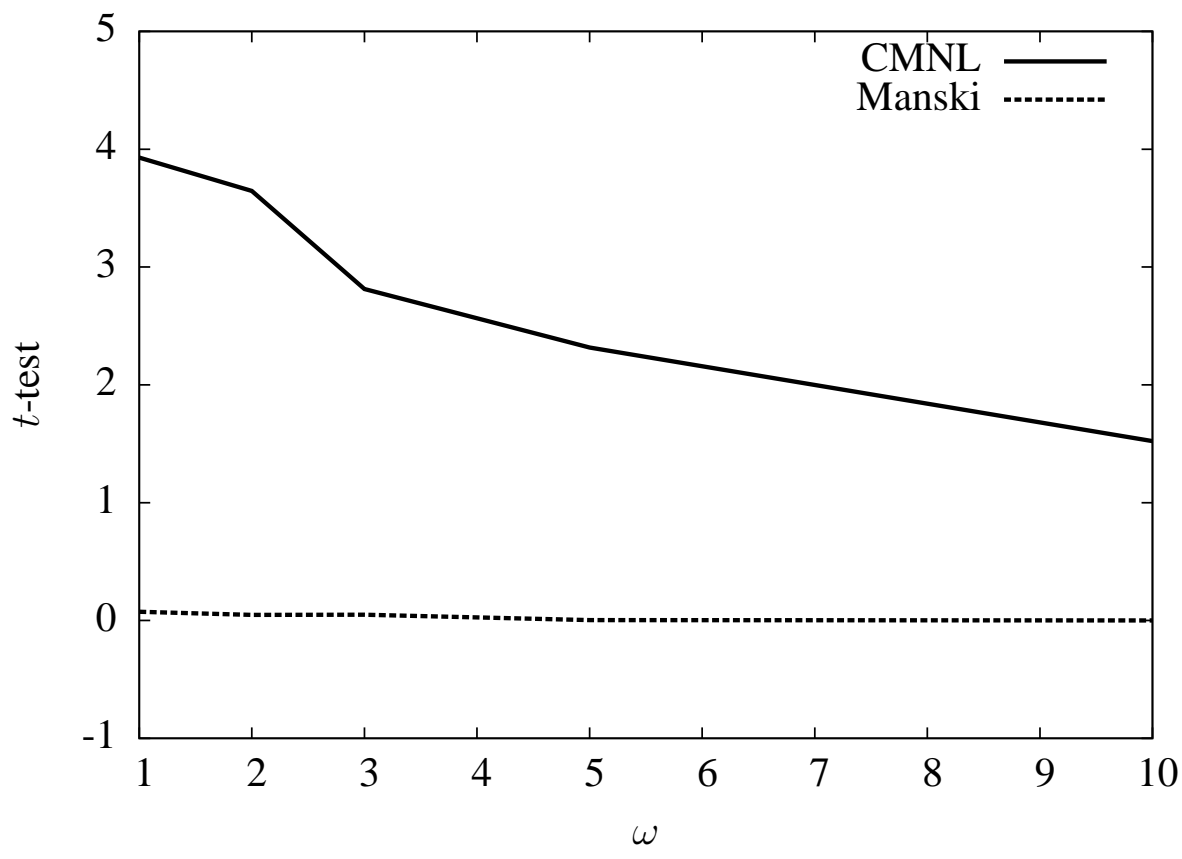

(b) Travel time

FIGURE $7 t$-statistics for the cost and time parameter over $\omega$ 


\section{REFERENCES}

[1] Martinez, F., F. Aguila, and R. Hurtubia, The Constrained Multinomial Logit Model: A SemiCompensatory Choice Model. Transportation Research Part B, Vol. 43, 2009, pp. 365-377.

[2] Manski, C., The structure of random utility models. Theory and Decision, Vol. 8, 1977, pp. 229-254.

[3] Swait, J. and M. Ben-Akiva, Incorporating random constraints in discrete models of choice set generation. Transportation Research Part B, Vol. 21, No. 2, 1987.

[4] Ben-Akiva, M. E. and B. Boccara, Discrete choice models with latent choice sets. International Journal of Research in Marketing, Vol. 12, 1995, pp. 9-24.

[5] Swait, J., Choice set generation within the generalized extreme value family of discrete choice models. Transportation Research Part B, Vol. 35, No. 7, 2001, pp. 643-666.

[6] Frejinger, E., M. Bierlaire, and M. Ben-Akiva, Sampling of Alternatives for Route Choice Modeling. Transportation Research Part B: Methodological, forthcoming, accepted for publication.

[7] Hauser, J., M. Ding, and S. Gaskin, Non-compensatory (and Compensatory) Models of Consideration-Set Decisions. In Proceedings of the Sawtooth Software Conference, Delray Beach, FL, 2009.

[8] Dieckmann, A., K. Dippold, and D. H., Compensatory versus noncompensatory models for predicting consumer preferences. Judgment and Decision Making, Vol. 4, 2009, pp. 200-213.

[9] Tversky, A., Elimination by aspects: a theory of choice. Psychological Review, Vol. 79, 1972, pp. 281-299.

[10] Gilbride, T. J. and G. M. Allenby, A Choice Model with Conjunctive, Disjunctive, and Compensatory Screening Rules. Marketing Science, Vol. 23, No. 3, 2004, pp. 391-406.

[11] Elrod, T., R. D. Johnson, and J. White, A new integrated model of noncompensatory and compensatory decision strategies. Organizational Behavior and Human Decision Processes, Vol. 95, No. 1, 2004, pp. 1 - 19.

[12] Cascetta, E. and A. Papola, Random utility models with implicit availability/perception of choice alternatives for the simulation of travel demand. Transportation Research Part C, Vol. 9, 2001, pp. 249-263.

[13] Cascetta, E., F. Pagliara, and K. Axhausen, The use of dominance variables in choice set generation. In Proceedings of the 11th World Conference on Transport Research, University of California at Berkeley, 2007.

[14] Gaudry, M. and M. Dagenais, The dogit model. Transportation Research Part B, Vol. 13, 1979, pp. 105-111.

[15] Bierlaire, M., K. Axhausen, and G. Abay, Acceptance of modal innovation: the case of the SwissMetro. In Proceedings of the 1st Swiss Transportation Research Conference, Ascona, Switzerland, 2001, www.strc.ch. 